\title{
The Impact of Traumatic Brain Injury on Neurocognitive Outcomes in Children: a Systematic Review and Meta-Analysis
}

\author{
Mark Sen Liang Goh 이, ${ }^{1}$ Dawn Shu Hui Looi, ${ }^{2}$ Jia Ling Goh, ${ }^{3}$ Rehena Sultana, ${ }^{4}$ \\ Sharon Si Min Goh, Jan Hau Lee, ${ }^{1,6}$ Shu-Ling Chong ${ }^{1,5}$
}

'Duke-NUS Medical School, Singapore

${ }^{2}$ Lee Kong Chian School of Medicine, Nanyang Technological University, Singapore

${ }^{3}$ Yong Loo Lin School of Medicine, National University of Singapore, Singapore

${ }^{4}$ Centre for Quantitative Medicine, Duke-NUS Medical

School, Singapore

${ }^{5}$ Department of Emergency Medicine, KK Women's and Children's Hospital, Singapore ${ }^{6}$ Children's Intensive Care Unit, KK Women's and Children's Hospital, Singapore

Correspondence to Dr Shu-Ling Chong, Department of Emergency Medicine, KK Women's and Children's Hospital, Singapore, Singapore; chong.shu-ling@kkh.com.sg

Received 8 September 2020 Revised 25 January 2021 Accepted 10 March 2021 Published Online First 31 March 2021

Check for updates

(C) Author(s) (or their employer(s)) 2021. No commercial re-use. See rights and permissions. Published by BMJ.

To cite: Goh MSL, Looi DSH, Goh JL, et al. J Neurol Neurosurg Psychiatry 2021:92:847-853.

\section{ABSTRACT}

Objective To assess the burden of paediatric traumatic brain injury (TBI) on neurocognition via a systematic review and meta-analysis.

Methods Studies that compared neurocognitive outcomes of paediatric patients with TBI and controls were searched using Medline, Embase, PsycINFO and Cochrane Central Register of Controlled Trials, between January 1988 and August 2019. We presented a random-effects model, stratified by TBI severity, time of assessment post injury and age.

Results Of 5919 studies, 41 (patients=3717) and 33 (patients $=3118$ ) studies were included for the systematic review and meta-analysis, respectively. Studies mostly measured mild TBI $(n=26$, patients=2888) at $0-3$ months postinjury $(n=17$, patients $=2502)$. At 0-3 months postinjury, standardised mean differences between TBI and controls for executive function were $-0.04\left(95 \% \mathrm{Cl}-0.14\right.$ to $\left.0.07 ; I^{2}=0.00 \%\right),-0.18$ $\left(95 \% \mathrm{Cl}-0.29\right.$ to $\left.-0.06 ; \mathrm{I}^{2}=26.1 \%\right)$ and $-0.95(95 \%$ $\mathrm{Cl}-1.12$ to $\left.-0.77 ; \mathrm{I}^{2}=10.1 \%\right)$ for mild, moderate and severe $\mathrm{TBI}$, respectively; a similar effect was demonstrated for learning and memory. Severe TBI had the worst outcomes across all domains and persisted $>24$ months postinjury. Commonly used domains differed largely from workgroup recommendations. Risk of bias was acceptable for all included studies.

Conclusion A dose-dependent relationship between TBI severity and neurocognitive outcomes was evident in executive function and in learning and memory. Cognitive deficits were present for TBIs of all severity but persisted among children with severe TBI. The heterogeneity of neurocognitive scales makes direct comparison between studies difficult. Future research into lesser explored domains and a more detailed assessment of neurocognitive deficits in young children are required to better understand the true burden of paediatric TBI.

\section{INTRODUCTION}

Globally, traumatic brain injury (TBI) affects three million children annually and is a leading cause of death and disability. ${ }^{1}$ Children with moderate to severe TBI experience deficits that persist into adulthood, affecting educational, employment and psychosocial outcomes. ${ }^{2} 3$ The Centers for Disease Control and Prevention identified poor understanding of the long-term consequences of paediatric TBI as an important gap resulting in inadequate care. ${ }^{4}$

Published studies exploring the neurocognitive effects of TBI vary in cognitive domains studied, severity of TBI assessed and timeframe for evaluation. $^{2} 5$ The most recent meta-analysis reviewed data from 1988 to 2007 and reported that children with moderate to severe TBI had significant impairments in various cognitive domains (intellectual function, processing speed, attention, fluency, inhibition, problem-solving and memory). ${ }^{2}$ Since this publication, the classification of cognitive domains has been updated by the Diagnostic and Statistical Manual of Mental Disorders, Fifth Edition (DSMV). ${ }^{6}$ To date, no systematic review or meta-analysis on TBI neurocognitive changes has used this new classification. Determining the burden of paediatric TBI on neurocognition using the updated cognitive domains will allow for a better understanding of this vulnerable population and facilitate future research in this area.

We aimed to summarise evidence from January 1988 to August 2019 and perform an updated systematic review and meta-analysis on neurocognitive outcomes of children with TBI. We purposed to compare them with controls (either healthy or with single-system orthopaedic injury) in the DSM-V cognitive domains: complex attention, executive function, learning and memory, language, perceptual-motor function, and social cognition. ${ }^{6}$ In addition, we hypothesised that there are worse outcomes in children with TBI compared with controls, for any severity (mild, moderate or severe) across one or more of the six defined cognitive domains, across any time-point.

\section{METHODS}

We followed the Preferred Reporting Items for Systematic Reviews and Meta-Analyses guidelines. This study is registered with PROSPERO (International Prospective Register of Systematic Reviews; CRD42020152680).

\section{Selection criteria}

Studies containing elements of the Population Exposure Comparator Outcome (PECO) format were searched. ${ }^{8}$ We defined the population as children 18 years or younger. We defined the exposure, TBI, as 'an alteration in brain function, or other evidence of brain pathology, caused 
by an external force' and graded by the Glasgow Coma Scale (GCS), where mild, moderate and severe TBI correspond to GCS scores 13-15, 9-12 and 3-8, respectively. ${ }^{9}{ }^{10}$ The control group comprised healthy children and those with single-system orthopaedic injury. Single-system orthopaedic injury refers to an isolated lower extremity orthopaedic injury without injuries to other body systems. ${ }^{11}$ We chose to include both control groups because the literature search revealed that many studies used either group as a comparator. Specifically, for hospital-based studies, single-system orthopaedic injuries were often used as the control group. When data from both control groups (healthy and single-system orthopaedic injury) were available, the healthy group was preferentially selected. Neurocognition is defined as 'the neural processes involved in the linking and processing of information, and comprises traditional neurocognitive domains found throughout the literature on $\mathrm{TBI}^{2}{ }^{6}$ We matched the neurocognitive outcomes to DSM-V cognitive domains: complex attention, executive function, language, learning and memory, perceptual-motor function, and social cognition. We defined the outcome as a change in neurocognition at the following timepoints: <24 hours, 0-3 months, 3-6 months, 6-12 months, 12-18 months, 18-24 months and >24 months. We included studies where the sample size for TBI in each severity group was at least 30 to prevent selection bias that may be present in small studies. $^{12}$ We included papers published from 1988 to $2019 .^{2}$ Only randomised controlled trials, cohort, case-control and cross-sectional studies were included. Systematic reviews were used to verify that relevant studies were included and were not used as part of the final set of studies analysed. Studies were excluded if they studied non-TBIs, were not peer-reviewed or not in English.

\section{Search strategy}

The literature search was conducted on 26 August 2019 via Medline, Embase, Cochrane Central Register of Controlled Trials and PsycINFO, in accordance with Cochrane Handbook guidelines. ${ }^{13}$ The search strategy was developed with input from a medical librarian and peer-reviewed by an independent search strategist. It was piloted in Medline using medical subject headings (MeSH) and refined using key articles found in preliminary searches. ${ }^{1415}$ The Medline (Ovid interface) search strategy was consistent across all databases (online supplemental table 1). Strategic search terms included paediatric, child, adolescent, traumatic brain injury, head injury, cognition and neurocognitive disorder. We hand-searched the reference list of systematic reviews and meta-analyses for relevant articles that were missed by the above search. We ensured there were no concurrent studies by searching PROSPERO, ClinicalTrials.gov, International Standard Randomised Controlled Trial Number registry, WHO International Clinical Trials Registry Platform and European Union Clinical Trials Register.

\section{Data collection}

Covidence (Veritas Health Innovation, Australia, V.1499) was used. Studies were first screened by title and abstract, with only PECO elements, and subsequently screened by full text where the eligibility criteria were applied. Reasons for each exclusion were recorded. Two reviewers independently screened each article during both stages. Conflicts were resolved by a third independent reviewer or through discussion. Neither of the reviewers were blinded to journal titles, study authors or institutions. Outcome estimates in the form of group data were sought. Two independent reviewers extracted the data using a standardised form on Microsoft Excel (Microsoft, USA). Studies that met the inclusion criteria but did not provide a detailed breakdown (eg, studies that comprised data spanning children and adults) were included if the authors provided the data after we contacted them. Differences in the data extracted were resolved through discussion. The standardised form included all variables of interest (online supplemental table 2). Measures used to assess neurocognition were each assigned a cognitive domain, based on DSM-V.

\section{Summary measures}

For the systematic review, we performed a narrative synthesis on the various outcome measures and their usage over time. Standardised mean differences (SMDs) were calculated between TBI and control groups for each study. Later, all SMDs were pooled using a DerSimonian and Laird method of inverse variance random-effects model. ${ }^{16}$ SMDs from individual studies and pooled results are presented in forest plots with 95\% CI. All statistical analyses were done using Comprehensive MetaAnalysis V.3 (Biostat, USA).

\section{Heterogeneity assessment}

The $\mathrm{I}^{2}$ statistics quantified heterogeneity between studies. ${ }^{17}$

\section{Publication bias}

Publication bias was evaluated using Egger's test and funnel plots. $^{18}$

\section{Data analysis}

Results from each outcome measure within each neurocognitive domain were analysed separately. Each domain-wise analysis was stratified by TBI severity and time-point. We used each assessment as a unique data point as each patient may be assessed using different measures at different time-points. To avoid withinsubject correlation, no overall pooling was done. Outcome estimates were then compared longitudinally within each TBI severity. Mean (SD) was extracted from all included studies. If studies reported the outcome as median (IQR or range), mean (SD) was estimated manually. ${ }^{19}$ We chose the most frequently used outcome measures (used three or more times in independent studies) in each cognitive domain and calculated the SMD effect sizes. We then stratified our data and presented this in a forest plot according to TBI severity and time-point.

\section{Meta-regression}

Meta-regression by age ( $0-5$ and $5-18$ years old $)^{2}$ was performed to evaluate differences in cognitive deficit between the two groups.

\section{Risk of bias}

We assessed the risk of bias (RoB) using the Scottish Intercollegiate Guidelines Network criteria for randomised controlled trials, cohort and case-control studies, and the National Heart, Lung, and Blood Institute tool for cross-sectional studies. ${ }^{20}{ }^{21} \mathrm{An}$ overall rating of high quality, acceptable, low quality or unacceptable was given to each study, evaluated by two independent assessors.

\section{Published protocol}

The protocol is available online. ${ }^{22}$ 


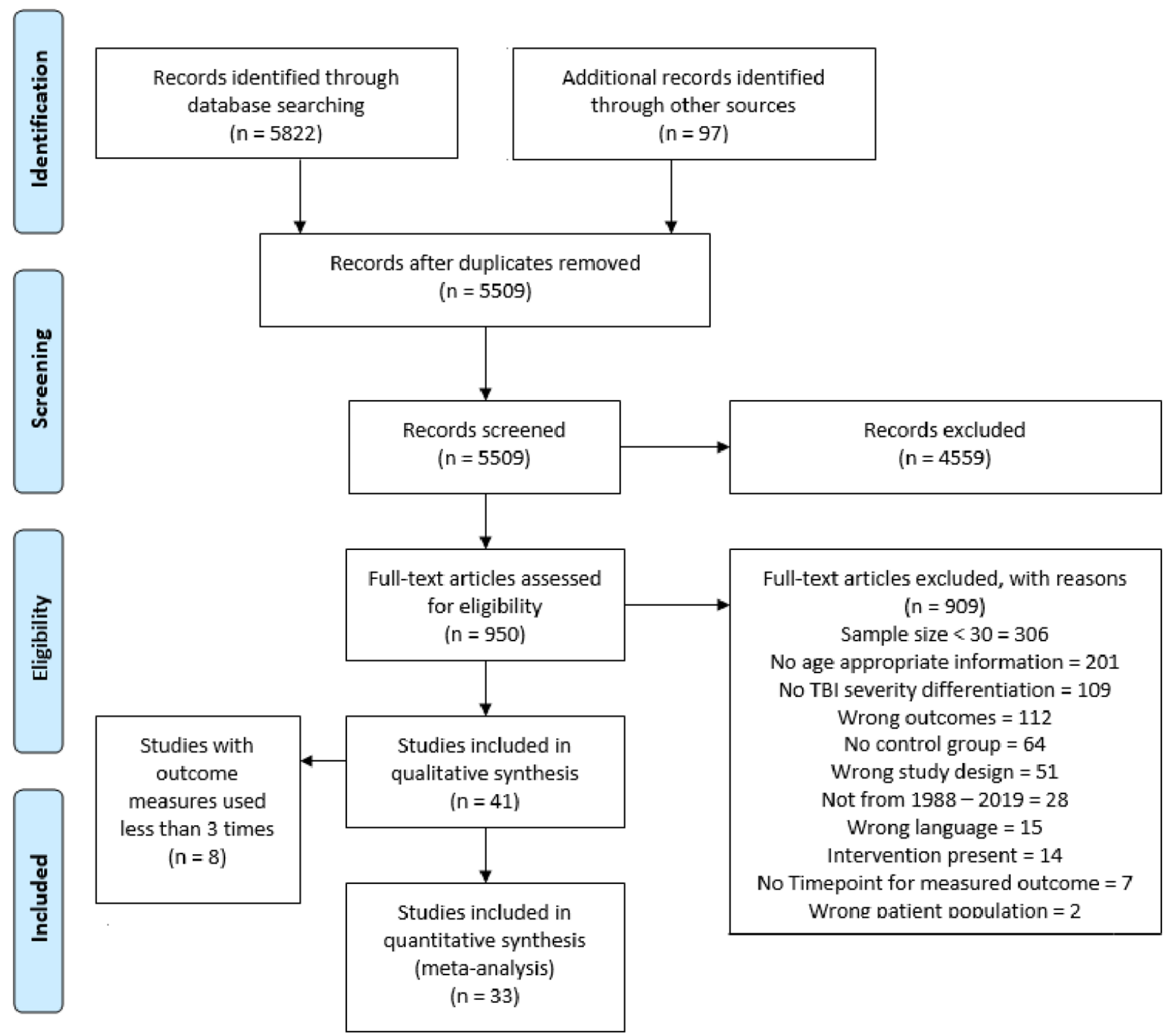

Figure 1 PRISMA flow chart. PRISMA, Preferred Reporting Items for Systematic Reviews and Meta-Analyses. TBI, traumatic brain injury.

\section{RESULTS}

Study selection

Among 5919 articles screened, 41 studies met the inclusion criteria and 33 were used in the meta-analysis (figure 1, online supplemental table 3 ). The excluded studies are listed in online supplemental table 4 .

\section{Study characteristics and design}

The 41 studies (total patients $=3717$ ) included in the systematic review were published between 1992 and 2018, with an increase in publications on learning and memory and on complex attention in 2012 (online supplemental figure 1). ${ }^{11 \text { s1-s40 }}$ Participants were recruited from the USA, Canada, Australia, Sweden, Switzerland and the Netherlands (online supplemental table 3). Twenty-four studies (58.5\%) measured mild TBI, ${ }^{11}$, s1-s4, s6, s12, s15-s17, s19, s21-s25, s28-s31, s33, s38-s40 seven studies (17.1\%) measured moderate TBI, ${ }^{\mathrm{s} 7}$, s14, s18, s26-s27, s36-s37 six studies (14.6\%) measured severe TBI, ${ }^{55}, \mathrm{~s} 8, \mathrm{~s} 9, \mathrm{~s} 20, \mathrm{~s} 32, \mathrm{~s} 34$ while two studies (4.9\%) measured both mild and severe $\mathrm{TBI}^{\mathrm{s} 10-\mathrm{s} 11}$ and two studies (4.9\%) measured both moderate and severe TBI. ${ }^{\text {s16, s35 }}$ There were 26 cohort studies, ${ }^{11 s 4, ~ s 10-s 15, ~ s 17-s 19, ~ s 21-s 25, ~ s 28-s 31, ~ s 34-s 36, ~ s 38-s 40 ~} 14$ cross-sectional studies $^{\mathrm{s} 1, \mathrm{~s} 2, \mathrm{~s} 5-\mathrm{s} 9, \mathrm{~s} 16, \mathrm{~s} 20, \mathrm{~s} 26-\mathrm{s} 27, \mathrm{~s} 32-\mathrm{s} 33, \mathrm{~s} 37}$ and 1 case-control study. ${ }^{\mathrm{s} 3}$

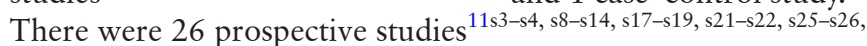

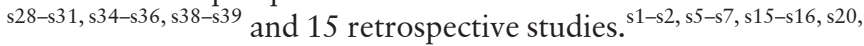
s23-s24, s27, s32-s33, s37, s40 Study setting was split between 26 hospitalbased, ${ }^{11 s 4, s 8-s 14, s 17-s 25, s 29, \mathrm{~s} 31, \mathrm{~s} 33-\mathrm{s} 36, \mathrm{~s} 38-\mathrm{s} 39} 11$ register-based, ${ }^{\mathrm{s} 2}, \mathrm{~s} 5-\mathrm{s} 7$, s15-s16, s26-s28, s30, s40 2 clinical-based $^{\mathrm{s} 32, \mathrm{~s} 37}$ and 2 referral-based. $^{\mathrm{s} 1 \text {, }}$ s3 There were different numbers of studies reporting on each time-point, the most frequently reported being 0-3 months for mild and moderate TBI and 6-12 months for severe TBI (online supplemental table 5). Seventy-two different outcome measures were used across all cognitive domains. The most common measures used in each domain are documented in table 1.

\section{Outcome measures reported}

Overall, $33(80.5 \%)$ of 41 studies (total patients=3118) employed outcome measures that were used three or more times in other studies and were included in the meta-analysis. ${ }^{11,}$ 


\begin{tabular}{|c|c|c|c|}
\hline Domain & Outcome measures & Publications $(\mathrm{n})^{*}$ & $\%$ of publications per domain \\
\hline \multirow[t]{3}{*}{ Complex attention $\left(\mathrm{n}^{*}=18\right)$} & Wechsler Intelligence Scale for Children & 7 & 38.9 \\
\hline & Immediate Post-Concussion Assessment and Cognitive Testing & 5 & 27.8 \\
\hline & Test of Everyday Attention for Children & 3 & 16.7 \\
\hline \multirow[t]{7}{*}{ Executive function $\left(n^{*}=27\right)$} & Wechsler Intelligence Scale for Children & 12 & 44.4 \\
\hline & Wechsler Abbreviated Scale of Intelligence & 4 & 14.8 \\
\hline & Delayed Alternation Test & 3 & 11.1 \\
\hline & Differential Ability Scales & 3 & 11.1 \\
\hline & Halstead-Reitan Neuropsychological Battery & 3 & 11.1 \\
\hline & Shape School & 3 & 11.1 \\
\hline & Wide Range Achievement Test & 3 & 11.1 \\
\hline \multirow[t]{2}{*}{ Language $\left(n^{*}=17\right)$} & Wechsler Intelligence Scale for Children & 5 & 29.4 \\
\hline & Wide Range Achievement Test & 3 & 17.6 \\
\hline \multirow[t]{3}{*}{ Learning and memory $\left(n^{*}=24\right)$} & California Verbal Learning Test & 7 & 29.2 \\
\hline & Immediate Post-Concussion Assessment and Cognitive Testing & 5 & 20.8 \\
\hline & Woodcock-Johnson Tests of Achievement & 3 & 12.5 \\
\hline \multirow[t]{3}{*}{ Perceptual-motor function $\left(n^{*}=10\right)$} & Wechsler Intelligence Scale for Children & 4 & 40.0 \\
\hline & Woodcock-Johnson Scales of Independent Behavior & 3 & 30.0 \\
\hline & Halstead-Reitan Neuropsychological Battery & 3 & 30.0 \\
\hline \multirow[t]{2}{*}{ Social cognition $\left(n^{*}=6\right)$} & Emotional and Emotive Faces Task & 5 & 83.3 \\
\hline & Ironic Criticism and Empathic Praise Task & 3 & 50.0 \\
\hline
\end{tabular}

${ }^{*}$ Number of publications.

s2-s10, s13-s14, s16-s23, s25-s31, s34-s35, s37-s40 The number of studies with common outcome measures within each cognitive domain was $14(77.8 \%)$ for complex attention, 20 (74.1\%) for executive function, $15(62.5 \%)$ for learning and memory, 6 (35.3\%) for language, 5 (50.0\%) for perceptual-motor function, and 5 $(83.3 \%)$ for social cognition. The common measures used for each domain were the Wechsler Intelligence Scale for Children (WISC) for executive function $(n=12,44.4 \%)$, complex attention $(n=7,38.9 \%)$, language $(n=5,29.4 \%)$ and perceptualmotor function $(\mathrm{n}=4,40.0 \%)$, the California Verbal Learning Test (CVLT) for learning and memory $(n=7,29.2 \%)$, and the Emotional and Emotive Faces Task (EEFT) for social cognition $(n=5,83.3 \%)$, where $n$ refers to the number of publications. A shift in common measures used was noted in 2008, while WISC was used consistently from 1992 through 2015 (online supplemental figure 2).

\section{Complex attention}

Estimates for complex attention showed worse outcomes in mild TBI at $<24$ hours (SMD $=-0.36,95 \% \mathrm{CI}-0.70$ to -0.01$)$, with resolution by $3-6$ months ( $\mathrm{SMD}=-0.13,95 \% \mathrm{CI}-0.35$ to 0.09 ) (online supplemental figure 3). Heterogeneity was substantial between studies with results at $<24$ hours $\left(\mathrm{I}^{2}=72.3 \%, \mathrm{p}=0.003\right)$; however, heterogeneity was low for studies examining outcomes at 3-6 months $\left(\mathrm{I}^{2}=33.7 \%, \mathrm{p}=0.197\right)$ (online supplemental table 6). The funnel plot showed no evidence of small-study effects, supported by Egger's test $(\mathrm{p}=0.948)$ (online supplemental figure 4). There were no estimates for moderate to severe TBI in the domain of complex attention.

\section{Executive function}

Pooled estimates for executive function showed a dose-dependent relationship between TBI severity and outcomes. At 0-3 months, the SMD was -0.04 (95\% CI -0.14 to 0.07$),-0.18$ (95\% CI -0.29 to -0.06$)$ and -0.95 (95\% CI -1.12 to -0.77$)$ for mild, moderate and severe TBI, respectively (figure 2). This pattern was similar at 6-12 months, 18-24 months and > 24 months. Patients with mild TBI showed resolution at $>24$ months $(\mathrm{SMD}=0.10$, $95 \%$ CI -0.04 to 0.24 ), while those with severe TBI continued to show worse outcomes at $>24$ months $(\mathrm{SMD}=-0.53,95 \%$ CI -1.25 to -0.18$)$. Heterogeneity was low at $0-3$ months for studies involving mild $\left(\mathrm{I}^{2}=0 \%, \mathrm{p}=0.948\right)$, moderate $\left(\mathrm{I}^{2}=26.1 \%\right.$, $\mathrm{p}=0.188)$ and severe $\left(\mathrm{I}^{2}=10.1 \%, \mathrm{p}=0.349\right)$ TBI and at $>24$ months for mild TBI $\left(\mathrm{I}^{2}=0 \%, \mathrm{p}=0.909\right)$ (online supplemental table 7). There was considerable heterogeneity at $>24$ months for severe TBI $\left(\mathrm{I}^{2}=78.5 \%, \mathrm{p}=0.031\right)$. Small-study effects were shown by funnel plot, supported by Egger's test $(\mathrm{p}<0.005)$ (online supplemental figure 5).

\section{Learning and memory}

Pooled estimates for learning and memory also showed a dosedependent relationship. At 0-3 months, SMD was $-0.21(95 \%$ CI -0.28 to -0.14$),-0.22(95 \%$ CI -0.41 to -0.03$)$ and -1.00 (95\% CI -1.18 to -0.83 ) for mild, moderate and severe TBI, respectively (online supplemental figure 6). In patients with mild TBI, this resolved by $>24$ months $(\mathrm{SMD}=-0.05,95 \%$ CI -0.25 to 0.15 ), while those with severe TBI showed worse outcomes at $18-24$ months $(\mathrm{SMD}=-0.79,95 \% \mathrm{CI}-0.97$ to $-0.60)$. Heterogeneity was low at $0-3$ months for studies of mild $\left(\mathrm{I}^{2}=30.7 \%, \mathrm{p}=0.078\right)$ and moderate $\left(\mathrm{I}^{2}=0 \%, \mathrm{p}=0.396\right) \mathrm{TBI}$, at $>24$ months for mild TBI $\left(\mathrm{I}^{2}=0 \%, \mathrm{p}=0.996\right)$, and at $18-24$ months for severe TBI $\left(\mathrm{I}^{2}=0 \%, \mathrm{p}=0.476\right)$ (online supplemental table 8). Heterogeneity was moderate at $0-3$ months for severe TBI $\left(I^{2}=56.0 \%, p=0.006\right)$. Small-study effects were shown by funnel plot, supported by Egger's test $(p<0.001)$ (online supplemental figure 7).

Language, perceptual-motor function and social cognition Overall pooled estimates for language, perceptual-motor function and social cognition showed no change with mild TBI at all time-points. Where data were available for severe TBI, deficits were shown across all time-points. Data for effect sizes, heterogeneity tests and publication bias can be found in online supplemental figures 8-13 and online supplemental tables 9-11. 
Group by TBI Grp

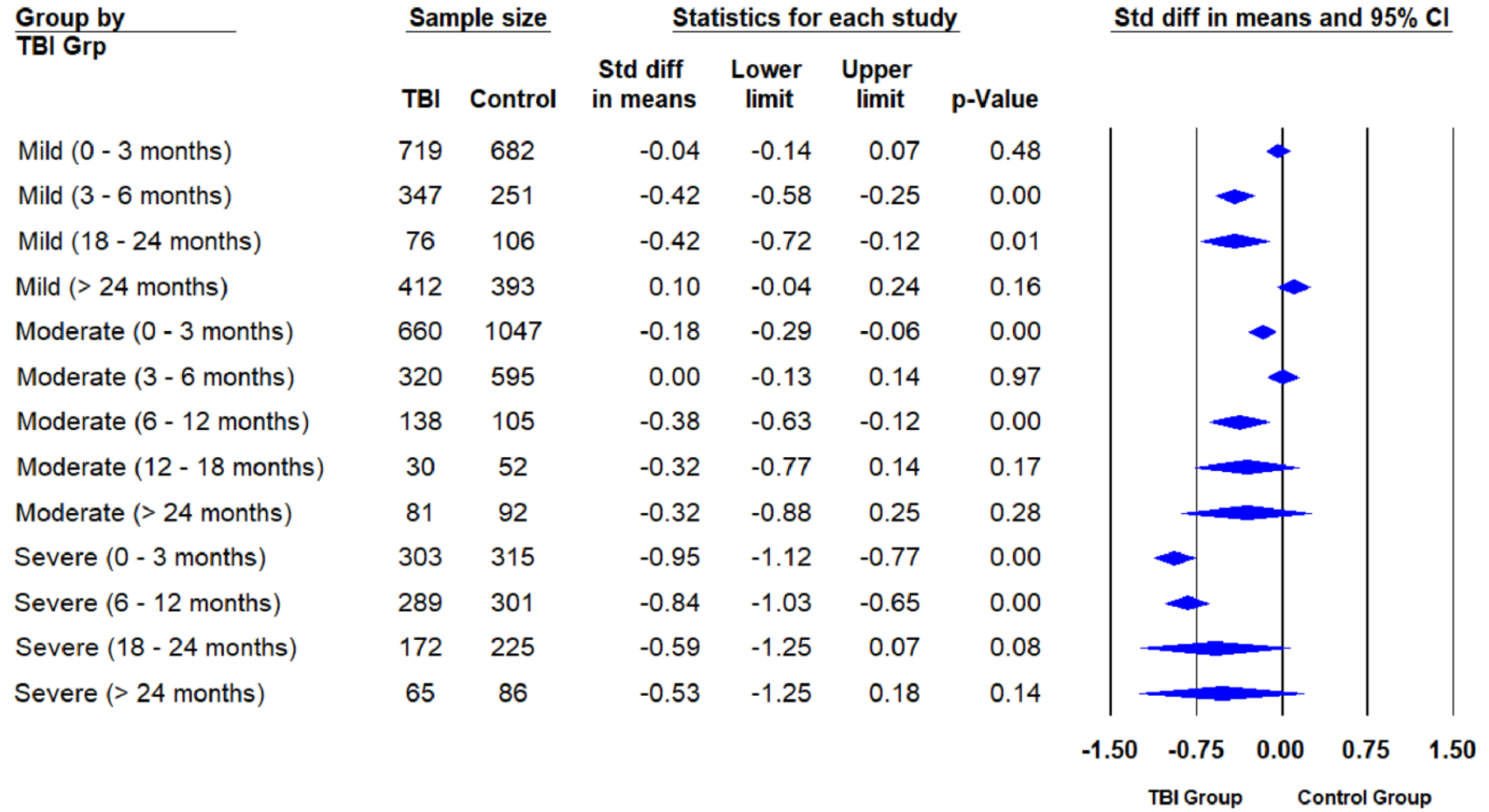

Std diff in means and $95 \% \mathrm{Cl}$

\section{Meta-regression by age}

Meta-regression was performed accounting for TBI severity and age. Data stratified by age (0-5 and 5-18 years old) were only available for executive function. Out of the 20 studies included in the meta-analysis for executive function, $2(10.0 \%)$ studies included patients $<5$ years old, ${ }^{\mathrm{s} 13, \mathrm{~s} 34} 16(80.0 \%)$ studies included patients $5-18$ years old, ${ }^{\mathrm{s} 4}, \mathrm{s6}-\mathrm{s} 8, \mathrm{~s} 15-\mathrm{s} 18$, s21-s22, s25, s30, s33 and $2(10.0 \%)$ studies did not report ages. ${ }^{\mathrm{s} 3}$, s19 Severe TBI (as compared with mild) and young age $<5$ years were both independently associated with poorer executive function scores $(-0.62,95 \%$ CI -0.77 to -0.47 ; and $-0.39,95 \%$ CI -0.56 to -0.21 ). Across all severities, young age $<5$ years was clearly associated with poorer executive function scores (online supplemental table 12).

\section{Risk of bias}

RoB assessments (online supplemental tables 13-15) showed that studies were generally well designed and directly applicable. All had a quality rating of 'fair'. However, three assessments (5.8\%) rated two cohort studies as not having comparable comparison groups. ${ }^{\text {s2, }}{ }^{s 40}$ In most studies, the demographics of the control groups were comparable with the TBI groups (online supplemental table 16). Three assessments (5.8\%) indicated that two cohort studies lacked a clear method of TBI assessment. ${ }^{\text {s13, s19 }}$
Thirteen assessments (46.4\%) over eight cross-sectional studies showed $<50 \%$ participation rate. ${ }^{\mathrm{s} 1-\mathrm{s} 2, \mathrm{~s} 5-\mathrm{s} 6, \mathrm{~s} 8, \mathrm{~s} 16, \mathrm{~s} 32-\mathrm{s} 33}$

\section{DISCUSSION}

In this systematic review and meta-analysis, we demonstrated that greater neurocognitive deficits are associated with greater injury severity. This dose-dependent relationship was evident in executive function and in learning and memory. These domains were well studied, contained common outcome measures and supported by sufficient studies to observe such a trend. Our results showed that the longer the time from injury, the less the cognitive deficit, in keeping with the anticipated course of recovery. ${ }^{23}{ }^{24}$ Neurocognitive deficits in executive function remain for extended periods, up to 18-24 months for mild TBI and $>24$ months for severe TBI.

\section{Impact of mild TBI findings}

Our findings challenge the notion that mild TBIs 'tend to resolve within weeks or days after the injury with complete resolution typically by 3 months', ${ }^{6}$ which guides mild TBI return-to-play protocols. Currently, many patients do not receive follow-up care despite remaining symptomatic. As such, formal follow-up may be beneficial for these patients. ${ }^{25}$ Mild TBI resulted in deficits in complex attention, executive function, and learning and memory. For the remaining domains of language, perceptualmotor function and social cognition, our review did not show worse outcomes in patients with mild TBI, across all time-points. This was similar to several small-group findings. ${ }^{26-28}$

\section{Impact of moderate and severe TBI findings}

For patients with moderate TBI, deficits in executive function and in learning and memory were found at $0-3$ months and resolved 
by 12-18 months. Children with severe TBI were better studied with domains spanning from executive function, language, learning and memory, to perceptual-motor function, with worse outcomes across all time-points. Although acute management of severe TBI is guided by established treatment algorithms, there is limited evidence to guide rehabilitation. ${ }^{29} 30$ Our study demonstrated a significant cognitive decline in patients with severe TBI across multiple domains, highlighting the urgent need for coordinated efforts to guide high-quality rehabilitation in children with severe TBI.

\section{Age stratification}

There were greater deficits in patients $0-5$ years old compared with patients 5-18 years old. The younger the child, the more vulnerable the developing brain is to trauma. ${ }^{31}$ The postnatal brain continues to undergo development and refinement, with sensitive periods of critical growth. ${ }^{32}$ Because brain maturation is not linear, TBI that occurs during periods of marked plasticity can severely blunt the developmental trajectories of these young children and devastate their potential, resulting in long-term physical, cognitive, psychological and emotional impairments. ${ }^{33}$ This confirms previous findings and highlights the importance of timely and effective TBI management and rehabilitation to mitigate the poorer prognosis in this vulnerable group. ${ }^{34}$ Postinjury, the young child's brain is constantly remodelling to encode new learning experiences. ${ }^{35}$ Key drivers of effective rehabilitation include training-based interventions that induce neuroplasticity, enriching the child's learning environment and enhancing interactions between the child and the caregivers. ${ }^{36}$ A multidisciplinary team is often needed to provide the injured child with physiotherapy, occupational therapy, and balance, psychological and cognitive therapy. ${ }^{37}$

\section{Discrepancy in outcome measures}

Excluding learning and memory, we found that the measures commonly used in the other domains had important discrepancies with the current Common Data Elements (CDE) workgroup recommendations (table 2 ). ${ }^{38}$

This highlights an important need for researchers to use common platforms. Considering the trend in measures used after

\begin{tabular}{|c|c|c|c|}
\hline McCauley et $a l^{38}$ & & Results & \\
\hline CDE Domains & $\begin{array}{l}\text { Core } \\
\text { measures }\end{array}$ & DSM-V Domains & Top measures \\
\hline $\begin{array}{l}\text { Language and } \\
\text { communication }\end{array}$ & WASI, CUSR & Language & WISC \\
\hline Social cognition & None & Social cognition & EEFT \\
\hline Executive functioning & D-KEFS & Executive function & WISC \\
\hline Memory & RAVLT, CVLT & Learning and memory & CVLT \\
\hline Motor/psychomotor & None & $\begin{array}{l}\text { Perceptual-motor } \\
\text { function }\end{array}$ & WISC \\
\hline Neuropsychological & WISC & Complex attention & WISC \\
\hline
\end{tabular}

impairment

$\begin{array}{ll}\text { Visual-spatial } & \text { None } \\ \text { General intellectual } & \text { WASI }\end{array}$

CDE, Common Data Elements; CUSR, Caregiver Unintelligible Speech Rating; CVLT, California Verbal Learning Test; D-KEFS, Delis-Kaplan Executive Function System;

EEFT, Emotional and Emotive Faces Task; RAVLT, Ray Auditory Verbal Learning Test WASI, Wechsler Abbreviated Scale of Intelligence; WISC, Wechsler Intelligence Scale for Children
2005 (online supplemental figure 2), the most common measures used by studies and CDE recommendations, we recommend that WISC be considered for measuring complex attention, executive function, language and perceptual-motor function, CVLT for learning and memory, and EEFT for social cognition. The use of CDE will help comparison of outcomes to improve long-term neurocognitive outcomes in children with TBI.

\section{Strengths and limitations}

Our study has several strengths. We employed cognitive domains that categorise neurocognitive deficits well. We pooled the most common outcome measures used per domain to better elucidate the true neurocognitive burden. A wide range of time-points allowed us to track trends consistently. We also attempted to stratify the neurocognitive outcomes by age. However, we recognise that this study has important limitations. First, the use of $\mathrm{MeSH}$ terms was limited as there were no $\mathrm{MeSH}$ terms related to social cognition. Second, the use of DSM-V cognitive domains excluded factors that may be indirectly related to cognition. Social aggression, for example, may not directly affect cognition, but may affect decision-making and social function. Third, the criteria of a minimum sample size of 30 excluded studies with small samples sizes. The purpose was to reduce bias from small studies, but we recognise that important data could be missed out in the process. Fourth, we chose to focus on common measures within each domain for the meta-analysis, assuming that these outcome measures are truly representative of each neurocognitive domain. ${ }^{38}$ Due to the lack of available data, we were also unable to account for the impact of important confounders, specifically that of deprivation on children who may not have the benefit of early education at baseline, nor received robust neurorehabilitation postinjury. This would be especially pertinent for children $<3$ years old with significant brain plasticity, since injuries at this age could devastate subsequent developmental trajectories. Lastly, a large number of the studies included in the meta-analysis were from North America and Australia. Future work with a global representation will be needed to confirm our findings.

Sources of heterogeneity in the included studies were from demographic, physiological, clinical and methodological factors. Demographic heterogeneity is from multiple background differences including parental and child education and psychological and emotional factors that make up a child's environment. Clinical heterogeneity results from different mechanisms of injury, severity, acute management and assessment of children with TBI. Performing a meta-analysis using the random-effects model, subgroup analysis or meta-regression is a way to account for heterogeneities. We used the random-effects model but could not perform a robust meta-regression or subgroup analysis due to non-availability or inadequate data on the above-mentioned factors. However, we managed to do meta-regression using age by categorising into age groups of $\geq 5$ or $<5$ years old.

\section{CONCLUSION}

This study highlights the true global burden of TBI on neurocognition and demonstrates a dose-dependent and time-dependent relationship between TBI severity and outcomes. Severe TBI causes long-term devastation in multiple cognitive domains. The younger the child, the greater the brain's neuroplasticity at the point of injury, and therefore the greater the devastation. An important discrepancy was shown between recommended measures for cognitive assessment and current scales used by researchers. Future research into lesser explored domains and 
assessment of neurocognitive deficits in young children are required to better understand the long-term consequences of paediatric TBI.

Acknowledgements We would like to thank Miss Peggy Fong, chief librarian at KK Women's and Children's Hospital, Singapore, for tirelessly helping us with the search strategy.

Contributors MSLG and S-LC coordinated the study. MSLG, DSHL and S-LC developed the search strategy and registered the protocol. MSLG, DSHL, JLG, SSMG and S-LC reviewed the studies. MSLG, DSHL, S-LC and JLG extracted data from the studies and conducted risk of bias assessments. RS conducted the statistical analyses. MSLG, RS, JHL and S-LC performed the data interpretation. MSLG drafted the manuscript. S-LC, MSLG, JHL, RS, DSHL, JLG and SSMG helped to revise the manuscript. All authors read and approved the final manuscript.

Funding This review is financially supported by the Academic Medicine Enhancing Training, Healthcare, Outcomes and Standards (ETHOS) Duke-NUS Medical Student Fellowship (AY2019-AY2020). Duke-NUS was not involved in the design of the protocol and analysis plan of the review and will not provide input on the interpretation of the results.

Competing interests None declared.

Patient consent for publication Not required.

Provenance and peer review Not commissioned; externally peer reviewed.

Data availability statement All data relevant to the study are included in the article or uploaded as supplementary information.

Supplemental material This content has been supplied by the author(s). It has not been vetted by BMJ Publishing Group Limited (BMJ) and may not have been peer-reviewed. Any opinions or recommendations discussed are solely those of the author(s) and are not endorsed by BMJ. BMJ disclaims all liability and responsibility arising from any reliance placed on the content. Where the content includes any translated material, BMJ does not warrant the accuracy and reliability of the translations (including but not limited to local regulations, clinical guidelines, terminology, drug names and drug dosages), and is not responsible for any error and/or omissions arising from translation and adaptation or otherwise.

\section{ORCID iD}

Mark Sen Liang Goh http://orcid.org/0000-0003-1183-314X

\section{REFERENCES}

1 Dewan MC, Mummareddy N, Wellons JC, et al. Epidemiology of global pediatric traumatic brain injury: qualitative review. World Neurosurg 2016:91:497-509.

2 Babikian T, Asarnow R. Neurocognitive outcomes and recovery after pediatric TBI: meta-analytic review of the literature. Neuropsychology 2009;23:283-96.

3 Zaloshnja E, Miller T, Langlois JA, et al. Prevalence of long-term disability from traumatic brain injury in the civilian population of the United States, 2005. J Head Trauma Rehabil 2008:23:394-400.

4 Haarbauer-Krupa JK, Glang A, Kurowski B. Report to Congress : the management of traumatic brain injury in children (N. C. for I. P. and C. (U. S.). D. of U. I. Prevention. \& C. for D. C. and P. (U.S.), Eds.), 2018. Available: https://stacks.cdc.gov/view/cdc/51852

5 Bardoni A, Galbiati S, Recla M, et al. Evolution of the cognitive profile in school-aged patients with severe TBI during the first 2 years of neurorehabilitation. Brain Inj 2013;27:1395-401.

6 American Psychiatric Association, DSM-5 Task Force.. Diagnostic and statistical manual of mental disorders: DSM-5TM (5th ed.). Arlington, VA, US: American Psychiatric Publishing, Inc, 2013: 624-7.

7 Moher D, Shamseer L, Clarke M, et al. Preferred reporting items for systematic review and meta-analysis protocols (PRISMA-P) 2015 statement. Syst Rev 2015;4:1.

8 Morgan RL, Whaley P, Thayer KA, et al. Identifying the PECO: a framework for Formulating good questions to explore the association of environmental and other exposures with health outcomes. Environ Int 2018;121:1027-31.

9 Menon DK, Schwab K, Wright DW, et al. Position statement: definition of traumatic brain injury. Arch Phys Med Rehabil 2010;91:1637-40.

10 Teasdale G, Jennett B. Assessment of coma and impaired consciousness. The Lancet 1974;304:81-4

11 Rieger $\mathrm{BP}$, Lewandowski LJ, Callahan JM, et al. A prospective study of symptoms and neurocognitive outcomes in youth with concussion vs orthopaedic injuries. Brain Inj 2013;27:169-78.
12 Dechartres A, Trinquart L, Boutron I, et al. Influence of trial sample size on treatment effect estimates: meta-epidemiological study. BMJ 2013;346:f2304.

13 Higgins JPT, Thomas J, Li T. Cochrane Handbook for systematic reviews of interventions version 6.1 (updated September 2020). Cochrane, 2020. Available: www.training. cochrane.org/handbook

14 Lumba-Brown A, Yeates KO, Sarmiento K, et al. Diagnosis and management of mild traumatic brain injury in children. JAMA Pediatr 2018;172:e182847.

15 D'Souza A, Mollayeva S, Pacheco N, et al. Measuring change over time: a systematic review of Evaluative measures of cognitive functioning in traumatic brain injury. Front Neurol 2019;10:353.

16 DerSimonian R, Laird N. Meta-Analysis in clinical trials. Control Clin Trials 1986;7:177-88.

17 Higgins JPT, Thompson SG. Quantifying heterogeneity in a meta-analysis. Stat Med 2002:21:1539-58.

18 Egger M, Davey Smith G, Schneider M, et al. Bias in meta-analysis detected by a simple, graphical test. BMJ 1997;315:629-34.

19 Wan X, Wang W, Liu J, et al. Estimating the sample mean and standard deviation from the sample size, median, range and/or interquartile range. BMC Med Res Methodol 2014;14:135.

20 Scottish Intercollegiate Guidelines Network (SIGN). SIGN 50: a guideline developer's handbook. Edinburgh: SIGN; 2015. (SIGN publication no. 50), November 2015. Available: http://www.sign.ac.uk

21 National Institutes of Health. Quality assessment tool for observational cohort and cross-sectional studies, 2014. Available: https://www.nhlbi.nih.gov/health-pro/ guidelines/in-develop/cardiovascular-risk-reduction/tools/cohort [Accessed 23 March 2020].

22 Looi DSH, Goh MSL, Goh SSM, et al. Protocol for a systematic review and metaanalysis of long-term neurocognitive outcomes in paediatric traumatic brain injury. BMJ Open 2020;10:e035513.

23 Sroufe NS, Fuller DS, West BT, et al. Postconcussive symptoms and neurocognitive function after mild traumatic brain injury in children. Pediatrics 2010;125:e1331-9.

24 Popernack ML, Gray N, Reuter-Rice K. Moderate-To-Severe traumatic brain injury in children: complications and rehabilitation strategies. J Pediatr Health Care 2015;29:e1-7.

25 Seabury SA, Gaudette Étienne, Goldman DP, et al. Assessment of follow-up care after emergency department presentation for mild traumatic brain injury and concussion. JAMA Netw Open 2018;1:e180210

26 Ewing-Cobbs L, Prasad MR, Swank P, et al. Social communication in young children with traumatic brain injury: relations with corpus callosum morphometry. Int I Dev Neurosci 2012;30:247-54

27 Stephens J, Salorio C, Denckla M, et al. Subtle motor findings during recovery from pediatric traumatic brain injury: a preliminary report. J Mot Behav 2017:49:20-6.

28 Liégeois FJ, Mahony K, Connelly A, et al. Pediatric traumatic brain injury: language outcomes and their relationship to the arcuate fasciculus. Brain Lang 2013;127:388-98.

29 Kochanek PM, Tasker RC, Bell MJ, et al. Management of pediatric severe traumatic brain injury. Pediatric Critical Care Medicine 2019;20:269-79.

30 Fuentes MM, Wang J, Haarbauer-Krupa J, et al. Unmet rehabilitation needs after hospitalization for traumatic brain injury. Pediatrics 2018;141:e20172859.

31 Keenan HT, Presson AP, Clark AE, et al. Longitudinal developmental outcomes after traumatic brain injury in young children: are infants more vulnerable than toddlers? J Neurotrauma 2019;36:282-92

32 Ismail FY, Fatemi A, Johnston MV. Cerebral plasticity: windows of opportunity in the developing brain. Eur J Paediatr Neurol 2017;21:23-48.

33 Li N, Yang Y, Glover DP, et al. Evidence for impaired plasticity after traumatic brain injury in the developing brain. J Neurotrauma 2014;31:395-403.

34 Karver CL, Wade SL, Cassedy A, et al. Age at injury and long-term behavior problems after traumatic brain injury in young children. Rehabil Psychol 2012;57:256-65.

35 Novak I, Morgan C. High-Risk follow-up: early intervention and rehabilitation. Handbook of Clinical Neurology, 2019.

36 Morris T, Gomes Osman J, Tormos Muñoz JM, et al. The role of physical exercise in cognitive recovery after traumatic brain injury: A systematic review. Restor Neurol Neurosci 2016:34:977-88

37 Huh JW, Raghupathi R. Therapeutic strategies to target acute and long-term sequelae of pediatric traumatic brain injury. Neuropharmacology 2019;145:153-9.

38 McCauley SR, Wilde EA, Anderson VA, et al. Recommendations for the use of common outcome measures in pediatric traumatic brain injury research. J Neurotrauma 2012;29:678-705. 